\title{
Inteligência emocional entre estudantes de enfermagem na pandemia de COVID-19: estudo reflexivo
}

\author{
Emotional intelligence among nursing students in the COVID-19 pandemic: reflective study \\ Inteligencia emocional en estudiantes de enfermería en la pandemia COVID-19: estudio reflexivo
}

Recebido: 24/02/2021 | Revisado: 03/03/2021 | Aceito: 04/03/2021 | Publicado: 14/03/2021

\author{
Mariane Valesca de Menezes Lacerda \\ ORCID: https://orcid.org/0000-0001-6331-3635 \\ Universidade Federal do Vale do São Francisco, Brasil \\ E-mail: lacerda.mariane.menezes@gmail.com \\ Simone Coelho Amestoy \\ ORCID: https://orcid.org/0000-0001-8310-2157 \\ Universidade Federal do Vale do São Francisco, Brasil \\ E-mail: simoneamestoy@hotmail.com
}

\begin{abstract}
Resumo
Objetivo: promover uma reflexão crítica sobre inteligência emocional entre estudantes de enfermagem na pandemia de COVID-19. Método: trata-se de um estudo teórico-reflexivo construído a partir análise da produção científica da temática e do arcabouço teórico da inteligência emocional. Resultados: a pandemia provocou mudanças nos processos de viver e tem causado repercussões danosas aos indivíduos e sociedades. Entre estudantes, público especialmente afetado, as principais situações estressoras têm sido as limitações financeiras, o ensino na modalidade remota, as incertezas relacionadas ao seu desempenho e às perspectivas de futuro profissional. Neste cenário, o desenvolvimento da inteligência emocional pode ser priorizado tendo em vista amenizar os reflexos psicossociais e emocionais negativos que têm afetado a atmosfera biopsicossocial das pessoas, estando associado, sobretudo, ao melhor modo de enfrentamento. Considerações finais: destaca-se a relevância de abordar a inteligência emocional de modo transversal, permeando os diversos cenários da formação, com vistas a fomentar seu desenvolvimento entre os estudantes de Enfermagem.
\end{abstract}

Palavras-chave: Inteligência emocional; Educação em enfermagem; Coronavírus; Ensino.

\begin{abstract}
Objective: to promote a critical reflection on emotional intelligence among nursing students in the COVID-19 pandemic. Method: it is a theoretical-reflective study built from an analysis of the scientific production of the theme and the theoretical framework of emotional intelligence. Results: the pandemic caused changes in the processes of living and has caused harmful repercussions to individuals and societies. Among students, especially affected audiences, the main stressful situations have been financial limitations, remote education and uncertainties related to their performance and prospects for their professional future. In this scenario, intelligence and emotional development can be prioritized in order to soften the negative psychosocial and emotional reflexes that have affected people's biopsychosocial atmosphere, being associated, above all, with the best way of coping. Final considerations: the relevance of addressing emotional intelligence in a transversal way is highlighted, permeating the various training scenarios, with a view to fostering its development among nursing students.
\end{abstract}

Keywords: Emotional intelligence; Nursing education; Coronavirus; Teaching.

\section{Resumen}

Objetivo: promover una reflexión crítica sobre la inteligencia emocional entre los estudiantes de enfermería en la pandemia COVID-19. Método: es un estudio teórico-reflexivo construido a partir de un análisis de la producción científica del tema y el marco teórico de la inteligencia emocional. Resultados: la pandemia provocó cambios en los procesos de vida y ha provocado repercusiones nocivas en los individuos y las sociedades. Entre los estudiantes, públicos especialmente afectados, las principales situaciones estresantes han sido las limitaciones económicas, la educación a distancia e incertidumbres relacionadas con su desempeño y perspectivas de futuro profesional. En este escenario se puede priorizar la inteligencia y el desarrollo emocional con el fin de mitigar los reflejos psicosociales y emocionales negativos que han afectado el ambiente biopsicosocial de las personas, asociándose, sobre todo, a la mejor forma de afrontamiento. Consideraciones finales: se destaca la relevancia de abordar la inteligencia emocional de forma transversal, permeando los distintos escenarios formativos, con miras a propiciar su desarrollo entre los estudiantes de enfermería.

Palabras clave: Inteligencia emocional; Educación en enfermería; Coronavirus; Enseñanza. 


\section{Introdução}

O conceito de inteligência emocional tem fomentado discussões entre pesquisadores de diversas áreas. Entres estes, destacam-se John D. Mayer e Peter Salovey que apresentaram a intersecção entre os conceitos de inteligência e emoção, definindo-a como habilidade de monitorar sentimentos e emoções em si e em outros indivíduos (Salovey \& Mayer, 1990). Assim, com esta aptidão, a pessoa é capaz de perceber e diferenciar emoções e usar as informações obtidas em sua análise para guiar pensamentos e ações.

Investigações sobre IE cresceram exponencialmente nos anos que se seguiram, sobretudo com as contribuições de Daniel Goleman à área. Em seu livro best seller intitulado Inteligência Emocional, publicado em 1996, o autor apresentou o termo Quociente Emocional (QE), equivalente a IE, em complementaridade à inteligência racional, denominada Quociente de Inteligência (QI). Na visão de Goleman, o arcabouço da Inteligência Emocional está ancorado em cinco componentes: a autoconsciência, que é a capacidade de refletir sobre as próprias emoções, mesmo em cenários conturbados; a autogestão que representa o controle sobre si mesmo na busca pelo equilíbrio emocional adaptativo; a automotivação refere-se ao usufruto da energia proveniente das emoções para enfrentamento das adversidades e alcance de metas; a empatia, por sua vez, consiste na percepção e compreensão de emoções alheias; e o gerenciamento de relacionamentos, resultado da confluência dos demais componentes, possibilita ampla compreensão, articulação e desenvoltura nas relações humanas (Goleman, 2012).

No atual cenário turbulento causado pela pandemia, a inteligência emocional pode ser adotada, com vistas a mitigar os reflexos danosos oriundos do estresse, medo da morte e de perder um familiar, bem como da ansiedade diante da modificação dos hábitos de vidas, gerados pelo distanciamento social. Neste sentido, a IE pode contribuir com o manejo de situações estressoras (Singh \& Sharma, 2012), tais como exigências da vida pessoal ou profissional e também eventos relacionados à crise desencadeada pelo novo Coronavírus (SARS-CoV-2) (World Health Organization [WHO], 2020a).

No que concerne ao contexto pandêmico da Coronavirus Disease 2019 (COVID-19), é pertinente pontuar que há repercussões para além dos indicadores de morbimortalidade. Diante da conjuntura desafiadora, as medidas implementadas para reduzir o pico de incidência de infecções, a exemplo das ações de distanciamento social e quarentena voluntária da população, com consequente fechamento de escolas e universidades, interferem, substancialmente na saúde mental e inteligência emocional das pessoas e comunidades. Todavia estas estratégias tendem a ser negligenciadas e até mesmo subestimadas, gerando descontinuidade nas ações de enfrentamento (Ferguson et al., 2020; Ornell, Schuch, Sordi, \& Kessler, 2020). Acrescenta-se, ainda, que no caso de nações caracterizadas pela desigualdade social e baixos níveis educacionais, como o Brasil, os parâmetros que possam estimar o impacto da pandemia na saúde mental e comportamento das pessoas são escassos (Ornell et al., 2020).

De acordo com a Organização das Nações Unidas para a Educação, a Ciência e a Cultura (UNESCO), mais de um bilhão de estudantes estão afastados das escolas e universidades em todo o mundo como resultado da pandemia. No Brasil, o número de estudantes afetados é superior a cinquenta milhões, dos quais 8,5 milhões cursam ensino superior (Organização das Nações Unidas para a Educação, a Ciência e a Cultura [UNESCO], 2020). A maioria das universidades ao redor do mundo substituiu o ensino presencial pela modalidade de Ensino Remoto Emergencial (ERE) por meio de plataformas online (Sundarasen et al., 2020), medida aqui efetivada pela Portaria No 544, de 16 de junho de 2020, do Ministério da Educação e Cultura (MEC) (Brasil, 2020a).

Diversos estudos têm se empenhando em conhecer e analisar as repercussões da pandemia e do ERE na saúde biopsicossocial dos estudantes universitários. Segundo Sundaresen et al. (2020), as principais situações estressoras para os acadêmicos no contexto de pandemia tem sido as limitações financeiras, o ensino na modalidade remota e, por conseguinte, as incertezas relacionadas ao seu desempenho e as perspectivas de futuro profissional. Ademais, foram verificadas, entre outras condições de saúde, proporções expressivas de estresse e ansiedade entre estudantes universitários durante a pandemia de COVID-19. 
Nesta perspectiva, a habilidade de inteligência emocional é fator determinante no modo de enfrentamento individual, uma vez que tem sido associada ao melhor desempenho acadêmico (Sánchez, Acedo, Herrera, \& García, 2016), a maior satisfação pessoal, bem-estar psicológico e social (Nages, Moreno, Chica, Pérez, \& Martínez, 2016) e às melhores habilidades de alunos para lidar com desafios emocionais, sobretudo na área da saúde e, em especial, na enfermagem (Lewis, Neville, \& Ashkanasy, 2017).

No que se refere à inteligência emocional entre estudantes de enfermagem, é notório o valor e a vastidão de estudos já conduzidos internacionalmente, todavia no contexto nacional, evidencia-se uma lacuna no conhecimento científico, tendo em vista a carência de estudos acerca do tema na área da Enfermagem. Diante do exposto, o estudo tem por objetivo promover uma reflexão crítica sobre inteligência emocional entre estudantes de enfermagem na pandemia de COVID-19.

\section{Metodologia}

Trata-se de um estudo qualitativo do tipo teórico-reflexivo construído a partir da produção científica acerca da gestão das emoções de estudantes de enfermagem na pandemia de COVID-19 e do arcabouço teórico da inteligência emocional. As reflexões emergidas foram tecidas com base na análise crítica da literatura e organizadas mediante eixos condutores da temática, acrescidos das percepções e argumentações das autoras (Pereira, Shitsuka, Parreira, \& Shitsuka, 2018).

Primeiramente, foi realizado um levantamento bibliográfico, por meio da consulta online nas bases de dados com vistas a conduzir o estudo foi realizada nos meses de agosto a novembro de 2020. Realizou-se busca por produções a partir da Biblioteca Virtual de Saúde (BVS), com os descritores "Inteligência Emocional" e "Educação em Enfermagem" da Medline via Pubmed por meio dos termos Medical Subject Headings (MeSH) "Emotional Intelligence" e "Education Nursing".

Referente à estratégia de combinação, adotou-se o operador booleano "AND”, haja vista que se trata de um assunto atual, não foi estipulado período de tempo de publicação das produções, sendo incluídos os artigos que estivessem disponíveis na íntegra, em português, inglês ou espanhol. Também foram selecionadas para reforçar o arcabouço teórico do estudo as obras de renomados pesquisadores da temática, entre eles Mayer, Salovey, Caruso e Goleman.

Por meio do procedimento de busca, foram identificadas 78 publicações com potencial para fundamentar este manuscrito. Após a avaliação dos títulos e resumos, 21 artigos foram considerados para leitura na íntegra e, contemplando os critérios de inclusão, puderam subsidiar a esta reflexão.

\section{Resultados e Discussão}

Com base na análise dos estudos e obras selecionadas construiu-se as duas categorias para a discussão dos achados, denominadas de Inteligência emocional: perspectivas teóricas e Inteligência emocional entre estudantes de enfermagem na pandemia de COVID-19.

\section{Inteligência emocional: perspectivas teóricas}

A compreensão da Inteligência Emocional requer o entendimento da emoção, experienciada cotidianamente pelos seres humanos. Salovey e Mayer (1990), em estudos pioneiros sobre o tema, definiram emoção como uma resposta complexa e organizada a estímulos internos e/ou externos, de significado positivo ou negativo para o indivíduo e cuja expressão perpassa subsistemas orgânicos, tais como psicológico, fisiológico, cognitivo, motivacional e experiencial.

Scherer (2005) define a emoção como um processo sincronizado entre os cinco componentes que estão imbuídos no estado emocional: componente cognitivo, responsável pelo processamento da informação pela avaliação dos acontecimentos e objetos; componente neurofisiológico, expressado por meio das manifestações e regulação corporal; componente motivacional, representado pelas tendências das ações a serem desencadeadas; componente de expressão motora, percebido nas expressões 
faciais e vocais, cuja função é comunicar reação e intenção de comportamento; e a experiência ou sentimento subjetivo, que monitora o estado do organismo em interação com o ambiente externo, ou seja, envolve a percepção e integração da emoção ao repertório individual.

Neste âmbito, cabe salientar que o conhecimento pleno acerca das emoções ainda não foi alcançado mesmo com os avanços tecno-científicos e a elucidação de fatos que denotam sua importância na evolução humana. Os achados ao longo das décadas permitem compreender os mecanismos das emoções a partir do sistema límbico e suas estruturas interconectadas, partindo da neuroanatomia e da neurofisiologia. Em geral, estas são responsáveis pela ligação de um pensamento ou evento específico e sensações internas e/ou estados viscerais, resultando em alterações psicofisiológicas e hemodinâmicas, tais como: musculares, cardiovasculares, oculares, endócrinas e exócrinas (Critchley, Eccles, \& Garfinkel, 2013).

Diferentes tipos de emoção levam o organismo a desenvolver variadas respostas, sobretudo para a ação imediata, com a qual está intimamente ligada. A sensação provocada com o surgimento de uma emoção consiste em processo inevitável e cuidadosamente coordenado, orquestrado pelo sistema límbico, uma das partes mais jovens e essenciais do cérebro humano. Assim, tomando como exemplo a raiva, a qual consiste em uma básica e elementar emoção, observam-se alterações como direcionamento do fluxo sanguíneo para as mãos, aceleração dos batimentos cardíacos e descargas hormonais, sobretudo de adrenalina preparando o indivíduo para uma resposta ágil e robusta, tipicamente visualizada neste estado emocional (Goleman, 2012).

Ainda, é salutar mencionar que a interpretação da emoção e diferenciação entre cada uma delas, bem como as tendências biológicas de ação, dependem de fatores, tais como vivências, memórias, cultura, biocomportamento, entre outros. Segundo Levenson (2014), os diferentes padrões de atividade somática e visceral desencadeados pelas emoções são detectados pelo cérebro e lá processados, ocorrendo então a tradução em sentimento, isto é, em experiência subjetiva. E são os sentimentos que, por sua vez, se associam às memórias, às experiências de vida, aos aspectos comportamentais, e motivam a regulação emocional, componente indispensável da Inteligência Emocional (Levenson, 2014; Mayer, Caruso, \& Salovey, 1999).

O grande passo foi dado em 1990, quando os pesquisadores John D. Mayer e Peter Salovey apresentaram a intersecção formal entre inteligência e emoção e propuseram a estrutura da Inteligência Emocional. Assim denominada, a IE surgiu como um conjunto de habilidades capazes de melhorar a expressão, avaliação e regulação eficaz das emoções em si e nos outros e, também, de utilizar sentimentos para orientar as ações, lançando mão da motivação e planejamento (Salovey \& Mayer, 1990).

Nos anos que se seguiram, a IE ganhou espaço no meio acadêmico e de igual modo se tornou popular entre o público geral, fenômeno encabeçado pelo autor Daniel Goleman a partir da publicação de "Emotional Intelligence". Neste livro, o autor reforça a compreensão de que a inteligência vai além das aptidões linguísticas e matemáticas, compartilhada, cada vez mais, por psicólogos e estudiosos. Para Goleman, a Inteligência Emocional é distinta, porém complementar ao Quociente de Inteligência (QI), e nasceu de reflexões baseadas nos aspectos que são essenciais ao bem-estar na vida e o sucesso nas relações humanas. Apresenta-se, então, em cinco domínios/aptidões: conhecer as próprias emoções, lidar com emoções, motivar-se, reconhecer emoções nos outros e lidar com relacionamentos (Goleman, 2012). Desta forma, surgem avanços na forma de compreender a inteligência emocional e sua facilidade de aplicá-la no cotidiano.

O modelo de Goleman, como vislumbrado, trouxe um diferencial por adicionar outras dimensões do indivíduo ao conceito de IE, tais como a motivação e características de personalidade. Por conseguinte, este acréscimo abriu espaço para outros modelos alternativos, a exemplo do modelo de Reuven Bar-On no ano de 1997, em que a chamada Inteligência EmocionalSocial se refere a habilidades emocionais e socias, intimamente relacionadas, que consistem em cinco grandes domínios: aptidões intrapessoais, aptidões interpessoais, adaptabilidade, administração do estresse e humor geral (Bar-On, 2006).

Devido ao surgimento dos modelos alternativos, como os dos mencionados autores Goleman e Bar-on, Mayer, Salovey e Caruso revisaram seus estudos e propuseram o modelo de quatro ramos de habilidades envolvidas na Inteligência Emocional, 
são elas: percepção e expressão de emoções, tanto em si como nos outros; assimilação das emoções no pensamento, que consiste na integração das emoções voltada a facilitação do pensamento para direcionar ações; compreensão das emoções, se refere ao entendimento dos significados das emoções; e o gerenciamento/gestão das emoções (GE) a partir da reflexão, que se trata da administração das emoções em si e nos outros com foco no crescimento pessoal (Mayer, Salovey, \& Caruso, 2000). O modelo proposto impulsionou a lógica de que o ser humano é dotado de capacidade de aprendizado constante a partir do monitoramento e da análise das emoções e sentimentos.

Pioneiros nos estudo sobre o tema, John Mayer e Peter Salovey em parceria com David Caruso aprofundaram-se no tema por meio de pesquisas teóricas e empíricas. Nesta direção, quase três décadas após a apresentação da primeira proposta da teoria da IE, os pesquisadores trouxeram atualizações teórico-práticas. Entre os principais achados, estava a descrição das sete diretrizes que guiam a teorização da IE, sendo elas: a inteligências emocional é uma habilidade mental; a inteligência emocional é melhor mensurada como uma habilidade; inteligência para a resolução de problemas não corresponde perfeitamente ao comportamento inteligente; para medir bem a IE, um teste deve abranger a área de resolução de problemas, fazendo amostragem adequada do assunto; os testes válidos devem ter conteúdo bem definido, que elicitem as habilidades mentais humanas relevantes; a inteligência emocional é uma inteligência ampla, assim está junto com outras 8 a 15 inteligências amplas no hall da inteligência geral; pertence ao grupo das inteligências que processam "informações quentes", assim denominadas por serem pessoais e significativas para um indivíduo (Mayer, Caruso, \& Salovey, 2016).

Ainda, no que tange ao modelo de quatro ramos de habilidades que fora anteriormente apresentado, surgiram algumas atualizações diante de novas evidências científicas. Sendo assim, outros tipos de raciocínios no leque de cada ramo foram adicionados e, por exemplo, o ramo "Compreensão de Emoções" que antes se referia a nomear emoções, saber suas causas e consequências e entender emoções complexas recebeu o acréscimo das habilidades de avaliação e previsão emocional, que também contribuem para a resolução de problemas. Ademais, apesar dos avanços, os autores indicaram que as habilidades mentais e cognitivas envolvidas na IE ainda precisam ser fielmente determinadas, sendo a construção e validação dos testes um caminho para tal, uma vez que o modelo de quatro ramos ainda recebe críticas pela imprecisão (Mayer et al., 2016).

Outra atualização refere-se à comparação entre os conceitos de inteligências social, pessoal e emocional, muitas vezes descritas como semelhantes ou iguais, mas apontadas no manuscrito como distintas por se dividirem em diferentes áreas de solução de problemas, embora todas pertencentes aos grupos das amplas e quentes inteligências, bem como serem igualmente equivalentes em complexidade, por envolver mecanismos de raciocínio cognitivo sofisticados. Ainda, neste manuscrito os autores propuseram que as evidências científicas apontam para a existência de regras para o raciocínio emocional, sendo seu conhecimento adaptativo (Mayer et al., 2016), o que pode indicar a melhora do desempenho de acordo com o aprendizado.

A inteligência emocional consiste em via dupla ao possibilitar o aprimoramento de relações intrapessoais, em menção a si próprio, e interpessoais, com outros indivíduos ou grupos (Amestoy, 2020). Desta forma, sua melhor compreensão facilitará outras dimensões, bem como o desempenho acadêmico, em especial, dos estudantes de enfermagem.

\section{Inteligência emocional entre os estudantes de enfermagem na pandemia de COVID-19}

Em 2019, um novo surto de doença respiratória foi deflagrado em Wuhan, cidade da China, sendo causado pelo novo coronavírus (SARS-CoV-2) (Brasil, 2020b). A transmissão por gotículas respiratórias caracterizou-se como principal meio de disseminação do vírus. Quanto aos sinais e sintomas, são mais comuns a febre $\left(\geq 37,8^{\circ} \mathrm{C}\right)$, tosse, dispneia, mialgia e fadiga. Além desses, são possíveis, embora menos prevalentes, a ocorrência de produção de escarro, dor de cabeça, hemoptise, diarreia, entre outros quadros clínicos que confluem para a necessidade de atendimento em Unidades de Terapia Intensiva (UTI) e para a alta mortalidade (Huang et al., 2020; Brasil, 2020c;). Em março de 2020 a emergência em saúde da COVID-19 foi elevada a patamar de pandemia pela OMS, diante do crescimento pungente do número de infectados e de países atingidos, reforçando a necessidade 
de empreender ações urgentes pelos países visando conter a expansão da doença e amenizar sua letalidade (World Health Organization [WHO], 2020b).

Diante do risco de contaminação em massa com as atividades presenciais, considerando o estado de pandemia e o potencial infeccioso do SARS-CoV-2, o Ministério da Educação (MEC) determinou a substituição das aulas presenciais por aulas em meios digitais por meio das portarias $\mathrm{N}^{\circ} 343$, de 17 de março de 2020 e $\mathrm{N}^{\circ}$ 544, de 16 de junho de 2016 (Brasil, 2020a).

Dentre as medidas adotadas para conter a contaminação, o isolamento social logo alcançou as instituições educacionais implicando a necessidade de adaptação das atividades presenciais para o Ensino Remoto Emergencial (ERE) (Saraiva, Traversini, \& Lockmann, 2020). Apesar de afetar milhares de pessoas, estudos que abordem o ERE na Enfermagem ainda são escassos na literatura nacional e internacional (Costa et al., 2020), embora já seja indicado que esta modalidade impõe fragilidades no que diz respeito, sobretudo, às práticas e estágios e à integração ensino-serviço-comunidade, alicerce da formação do enfermeiro (LIRA et al., 2020). Tais desafios geram a necessidade de reflexões acerca das adequações necessárias para a continuidade do ensino e suas consequências para os estudantes.

Como já mencionado, costumam ser relatadas diversas implicações à saúde mental de estudantes durante a graduação, em especial naqueles dos cursos da saúde. Na atual conjuntura, estudantes se tornam expostos à fatores estressores adicionais, é necessário estar atento a essas situações. Estudo realizado com estudantes de enfermagem em Israel, no contexto de pandemia, evidenciou prevalência de ansiedade de 55,9\% e os principais problemas associados foram a incerteza econômica, medo da infecção em si e em familiares, necessidade de prestar cuidados a familiares e desafios para lidar com a educação à distância (Savitsky, Findling, Ereli, \& Hendel, 2020).

Em virtude de tal cenário, é essencial a adoção de sensibilidade que permita reconhecer a pandemia e suas repercussões como fenômenos psicossociais, sobretudo, considerando que indivíduos vivem situações complexas em seus espaços de vida, as quais envolvem um mosaico de sentimentos, tais como frustração, raiva e ressentimento (Sundarasen et al., 2020). Destarte, em meio ao cenário excepcional de pandemia, ser capaz de gerir emoções é parte do processo de cuidado de si mesmo e, por conseguinte, do outro, bem como auxilia o indivíduo a adotar melhores estratégias de enfrentamento de situações caóticas e desenvolver maior habilidade de adaptação e inovação na vida em sua completude (Amestoy, 2020).

$\mathrm{O}$ crescimento de discussões acerca da Inteligência Emocional fomentou o reconhecimento da gestão emocional para o desenvolvimento dos indivíduos, partindo do fato que a inteligência não é exclusivamente cognitiva, uma vez que envolve aspectos subjetivos e, por conseguinte, comportamentais (Goleman, 2012). Trazendo a discussão para o âmbito do trabalho, em especial para a enfermagem, é pertinente destacar a dicotomia entre tecno-cientificismo e o componente emocional. Entretanto, um movimento global tem colocado os atributos da Inteligência Emocional como essenciais para a enfermagem, seja no processo formativo ou no exercício profissional, conforme evidenciam estudos (Kaya, Şenyuva, \& Bodur, 2017; Sharon, \& Grinberg, 2018; Guo, Yin, Wang, Nie, \& Wang, 2019).

Por manter numerosas relações intra e interpessoais ora consigo mesmo, outrora com colegas e clientes, o enfermeiro necessita lidar com emoções, assim como ser dotado de sensibilidade e empatia, ser capaz de direcionar pensamentos e canalizar energia, motivar-se diante de desafios, interpretar linguagem não verbal, solucionar problemas e tomar decisões de maneira eficaz e consciente, dentre outras habilidades inerentes ao equilíbrio emocional (Goleman, 2012). Neste contexto, é salutar indicar que segundo Smith, Profetto-McGrath, e Cummings (2009), ser inteligente emocionalmente faz parte da natureza da enfermagem enquanto profissão e é vital na prática.

Assim, considerando o compromisso ético-social da profissão e a responsabilidade das instituições de ensino no processo educativo, fica evidente a necessidade de discutir esse tema e prover meios para o desenvolvimento do equilíbrio emocional ainda na graduação por meio da criação de oportunidades no currículo que sejam adequadas para a transferência desse conhecimento aos estudantes de enfermagem, com vistas ao aprimoramento de sua competência clínica e bem estar 
pessoal/profissional.

Nesta perspectiva, a produção científica que trata da intersecção entre IE e enfermagem, em especial na alçada da educação, aponta que, principalmente, o estudante de enfermagem precisa compreender e se apropriar da natureza emocional da profissão. Ainda, indica que este é o caminho para que o discente possa se preparar para a prática cotidiana, uma vez que as habilidades emocionais são requeridas tanto para ofertar cuidado de qualidade às pessoas, quanto para gerenciar situações conflituosas em diferentes ambientes de trabalho (Smith, Profetto-Mcgrath, \& Cummings, 2009). Isto posto, considera-se essencial abordar os componentes intra e interpessoal na formação, para além dos elementos técnicos (Santos, Almeida, \& Lemos, 1990). Neste sentido, o desenvolvimento de habilidades como autoconsciência, a autogestão, a automotivação, a empatia e gerenciamento de relacionamentos podem ser discutidas e dentro do possível, fomentadas na formação em Enfermagem, com vistas a auxiliar no aprimoramento da inteligência emocional dos estudantes.

No tocante ao processo de ensino-aprendizagem, a organização curricular prevê a formação em enfermagem primeiro orientada por disciplinas teóricas e, nos semestres mais avançados, predominam as práticas e estágios, período em que os estudantes demonstram sentir maior necessidade de controle emocional (Santos et al., 1990). Ademais, no decorrer da formação superior, estudantes experienciam uma variedade de eventos negativos, tais como ansiedade, estresse e depressão relacionados a fatores acadêmicos, cuja prevalência se concentra entre alunos da área da saúde (Ariño \& Bardagi, 2018; Carvalho, Bertolini, Milani, \& Martins, 2015). Esta realidade reafirma a importância das habilidades emocionais frente à necessidade de assegurar o bem-estar dos estudantes e de preparar os futuros profissionais da enfermagem, considerando os benefícios já citados (SÁNCHEZ et al., 2016; Nages et al., 2016; Lewis, Neville, \& Ashkanasy, 2017; Kaya, Şenyuva, \& Bodur, 2017; Sharon \& Grinberg, 2018; Guo et al., 2019; Amestoy, 2020).

Apesar de ser vista como essencial às habilidades e competências profissionais dos estudantes e enfermeiros, Ranjbar (2015) aponta que a inteligência emocional ainda não se estabeleceu como fator chave nos currículos em enfermagem, situação que tem preocupado sistemas de educação em enfermagem em todo mundo (Codier \& Odell, 2014).

Com base no exposto, destaca-se a necessidade de investimentos no desenvolvimento da inteligência emocional entre os estudantes de enfermagem, o qual poderá ocorrer de forma transversal, ao longo da graduação. Além disso, está habilidade relacional é necessária ainda mais, para auxiliar no enfrentamento de possíveis situações desgastantes emocionalmente, vivenciadas na pandemia causada pelo novo Coronavírus.

\section{Considerações Finais}

Diante de tantos percalços gerados pela pandemia de COVID-19, sinaliza-se que investimentos no desenvolvimento da inteligência emocional poderá ser uma estratégia valiosa na formação de futuros enfermeiros, considerando o momento crítico e turbulento vivenciado na saúde pública mundial. Sugere-se investimentos no desenvolvimento da inteligência emocional no decorrer da graduação em enfermagem, sendo abordada de modo transversal nos componentes curriculares.

Quanto à limitação do estudo convém destacar a busca da produção científica em duas bases de dados. Desta forma, recomenda-se que novas pesquisas possam ser realizadas em outras bases, com vistas a agregar maior aprofundamento da temática. Além disso, sugere-se o desenvolvimento de pesquisas com objetivo de conhecer os escores de Inteligência Emocional dos estudantes de enfermagem do Brasil e sua relação com variáveis sociodemográficas. Ainda, é interessante a realização de trabalhos que visem construir estratégias de fomento ao aperfeiçoamento da Inteligência Emocional entre alunos do ensino superior.

O estudo contribuiu para tecer reflexões acerca da relevância de abordar e desenvolver a inteligência emocional no cenário da graduação e de forma transversal, permeando os diversos cenários da formação do Curso de Enfermagem. 


\section{Referências}

Amestoy, S. C. (2020). Inteligência emocional: habilidade relacional para o enfermeiro-líder na linha de frente contra o novo coronavírus. J. nurs. health, 10 (n.esp.), e20104016.

Ariño, D. O., \& Bardagi, M. P. (2018). Relação entre Fatores Acadêmicos e a Saúde Mental de Estudantes Universitários. Psicol. pesq. [online], 12 (3), 44-52. Bar-On, R. (2006). The Bar-On model of emotional-social intelligence (ESI). Psicothema, 18, 13-25.

Carvalho, A. E., Bertolini, S. M. M. G., Milani, R. G., \& Martins, M. C. (2015). Índice de ansiedade em universitários ingressantes e concluintes de uma instituição de Ensino Superior. Ciência, Cuidado e Saúde, 14 (3), 1290-98.

Codier, E., \& Odell, E (2014). Measured emotional intelligence ability and grade point average in nursing students. Nurse Education Today, 34 (4), 608-12.

Costa, R., Lino, M. M., Souza, A. I. J., Lorenzini, E., Fernandes, G. C. M., Brehmer, L. C. F., Vargas, M. A. O., Locks, M. O. H., \& Gonçalves, N. (2020). Ensino de enfermagem em tempos de covid-19: como se reinventar nesse contexto? Texto contexto - enferm., 29 , e20200202.

Critchley, H. D., Eccles, J., \& Garfinkel, S. N. (2013). Interaction between cognition, emotion, and the autonomic nervous system. In: Buijs, R. M., \& Swaab, D. F. Handbook of clinical neurology, 117(6), 59-77, Elsevier.

Ferguson, N. M., Laydon, D., Nedjati-Gilani, G., Imai, N., Ainslie, K., Baguelin, M., Bhatia, S., Boonyasiri, A., Cucunubá, Z., Cuomo-Dannenburg, G., Dighe, A., Dorigatti, I., Fu, H., Gaythorpe, K., Green, W., Hamlet, A., Hinsley, W., Okell, L. C., Elsland, S. V., Thompson, H., Verity, R., Volz, E., Wang, H., Wang, Y., Walker, P. G. T., Walters, C., Winskill, P., Whittaker, C., Donnelly, C. A., Riley, S., \& Ghani, A. C. (2020). Report 9: impact of non-pharmaceutical interventions (NPIs) to reduce COVID19 mortality and healthcare demand (relatório 9 do Imperial College COVID-19 Response Team). https://www.imperial.ac.uk/media/imperial-college/medicine/mrc-gida/2020-03-16-COVID19-Report-9.pdf

Foster, K., Fethney, J., McKenzie, H., Fisher, M., Harkness, E., \& Kozlowski, D. (2017). Emotional intelligence increases over time: A longitudinal study of Australian pre-registration nursing students. Nurse Education Today, 55, 65-70.

Goleman, D. (2012). Inteligência emocional: a teoria revolucionária que define o que é ser inteligente.: Objetiva.

Guo, M., Yin, X., Wang, C., Nie, L., \& Wang, G. (2019). Emotional intelligence a academic procrastination among junior college nursing students. J Adv Nurs., $75(11), 2710-18$.

Huang, C., Wang, Y., Li, X., Ren, L., Zhao, J., Hu, Y., Zhang, L., Fan, G., Xu, J., Gu, X., Cheng, Z., Yu, T., Xia, J., Wei, Y., Wu, W., Xie, X., Yin, W., Li, G., Liu, M., Xiao, Y., Gao, H., Guo, L., Xie, J., Wang, G., Jiang, R., Gao, Z., Jin, Q., Wang, J., \& Cao, B. (2020). Clinical features of patients infected with 2019 novel coronavirus in Wuhan, China. Lancet, 395, 497-506.

Kaya, H., Şenyuva, E., \& Bodur, G. (2017). Developing critical thinking disposition and emotional intelligence of nursing students: a longitudinal research. Nurse Education Today, 48, 72-7.

Levenson, R. W. (2014). The autonomic nervous system and emotion. Emotion Review, 6 (2), 100-112.

Lewis, G. M., Neville, C., \& Ashkanasy, N. M. (2017). Emotional intelligence and affective events in nurse education: A narrative review. Nurse Education Today, 53, 34-40.

Lira, A. L. B. C., Adamy, E. K., Teixeira, E., \& Silva, F. V. (2020). Nursing education: challenges and perspectives in times of the COVID-19 pandemic. Rev Bras Enferm, 73 (suppl. 2), 1-6.

Mayer, J. D., Caruso, D. R., \& Salovey, P. (1999). Emotional intelligence meets traditional standards for an intelligence. Intelligence, 27 (4), $267-298$.

Mayer, J. D., Salovey, P., \& Caruso, D. R. (2000). Models of emotional intelligence. In: Sternberg, R. J. Handbook of intelligence (Cap. 18, p. 396-420): Cambridge University Press.

Mayer, J. D., Salovey, P., \& Caruso, D. R. (2016). The Ability Model of Emotional Intelligence. Emotion Review, 8 (4), $290-300$.

Ministério da Educação. (2020a). Portaria $n^{\circ} 544$, de 16 de junho de 2020. (2020). Dispõe sobre a substituição das aulas presenciais por aulas em meios digitais, enquanto durar a situação de pandemia do novo coronavírus - Covid-19, e revoga as Portarias MEC $\mathrm{n}^{\circ} 343$, de 17 de março de 2020 , $\mathrm{n}^{\circ} 345$, de 19 de março de 2020, e n 473 , de 12 de maio de 2020. https://www.in.gov.br/en/web/dou/-/portaria-n-544-de-16-de-junho-de-2020-261924872

Ministério da Saúde (2020c). Secretaria de Ciência, Tecnologia, Inovação e Insumos Estratégicos em Saúde. Diretrizes para diagnóstico e tratamento da COVID19. https://portalarquivos.saude.gov.br/images/pdf/2020/May/08/Diretriz-Covid19-v4-07-05.20h05m.pdf

Ministério da Saúde. (2020b). Secretaria de Atenção Especializada à Saúde. Protocolo de manejo clínico da Covid-19 na Atenção Especializada. https://portalarquivos.saude.gov.br/images/pdf/2020/April/14/Protocolo-de-Manejo-Cl--nico-para-o-Covid-19.pdf

Nages, J. L. S., Moreno, L. A., Chica, O. D., Pérez, E. E., \& Martínez, A. R. (2016). Inteligencia Emocional y Bienestar II: reflexiones, experiencias profesionales e investigaciones: Ediciones Universidad San Jorge.

Navarro, M. Q., \& Navarro, M. Q. (2020). Inteligencia emocional y estrés académico em estudiantes de enfermería. Ciencia y Enfermería, 26 (3), 1-9.

Ornell, F., Schuch, J. B., Sordi, A. O., \& Kessler, F. H. P. (2020). "Pandemic fear" and COVID-19: mental health burden and strategies. Brazilian Journal of Psychiatry. Braz J Psychiatry., 42 (3), 232-235.

Pereira, A. S., Shitsuka, D. M., Parreira, F. J., \& Shitsuka, R. (2018). Metodologia da pesquisa científica. UFSM. https://repositorio.ufsm.br/bitstream/handle/1/ 15824/Lic_Computacao_Metodologia-Pesquisa-Cientifica.pdf?sequence=1 
Research, Society and Development, v. 10, n. 3, e22410313269, 2021

(CC BY 4.0) | ISSN 2525-3409 | DOI: http://dx.doi.org/10.33448/rsd-v10i3.13269

Ranjbar, H. (2015). Emotional intelligence training: A necessity for nursing education curriculum. Nurse Educ Today., 35 (11), 1053.

Salovey, P., \& Mayer, J. D. (1990). Emotional intelligence. Imagination, Cognition, and Personality, 9 (3), $185-211$.

Sánchez, I. D. R., Acedo, M. A. D., Herrera, S. S., \& García, M. L. B. (2016). La inteligencia emocional en estudiantes universitarios: diferencias entre el grado de maestro en educación primaria y los grados en ciencias. International Journal of Developmental and Educational Psychology INFAD Revista de Psicología, $2(1), 51-62$.

Santos, L. M., Almeida, F. L., \& Lemos, S. C. (1999). Inteligência Emocional: testando a enfermagem do futuro. R. Bras. Enferm., 52 (3), 401-12.

Saraiva, K., Traversini, C., \& Lockmann. K. (2020). A educação em tempos de COVID-19: ensino remoto e exaustão docente. Práxis Educativa, 15, n.e2016289, $1-24$.

Savitsky, B., Findling, Y., Ereli, A., \& Hendel, T. (2020). Anxiety and coping strategies among nursing students during the covid-19 pandemic. Nurse Educ Today, 46 (102809), 1-7.

Scherer, K. R. (2005). What are emotions? And how can they be measured? Social Science Information, 44 (4), 695-729.

Sharon, D., \& Grinberg, K. (2018). Does the level of emotional intelligence affect the degree of success in nursing studies? Nurse Education Today, 64, 21-26.

Singh, Y., \& Sharma, R. (2012). Relationship between general intelligence, emotional intelligence, stress levels and stress reactivity. Annals of Neurosciences, $19(3), 107-11$.

Smith, K. B., Profetto-Mcgrath, J., \& Cummings, G. G. (2009). Emotional intelligence and nursing: An integrative literature review. Int J Nurs Stud, 46 (12), 1624-36.

Štiglic, G., Cilar, L., Novak, Ž., Vrbnjak, D., Stenhouse, R., Snowden, A., \& Pajnkihar, M. (2018) Emotional intelligence among nursing students: Findings from a cross-sectional study. Nurse Education Today, 66, 33-38.

Sundarasen, S., Chinna, K., Kamaludin, K., Nurunnabi, M., Baloch, G. M., Khoshaim, H. B., Hossain, S. F. A., \& Sukayt, A. (2020). Psychological Impact of COVID-19 and Lockdown among University Students in Malaysia: Implications and Policy Recommendations. Int. J. Environ. Res. Public Health, 17 (17), 6206

United Nations Educational, Scientific and Cultural Organization [UNESCO] (2020). Education: From disruption to recovery. https://en.unesco.org/covid19/educationresponse

World Health Organization [WHO] (2020a). Mental health and psychosocial considerations during COVID-19 outbreak. Geneva: WHO.

World Health Organization [WHO] (2020b). WHO Director-General's opening remarks at the media briefing on COVID-19 - 11 March 2020. https://www.who.int/director-general/speeches/detail/who-director-general-s-opening-remarks-at-the-media-briefing-on-covid-19---11-march-2020 